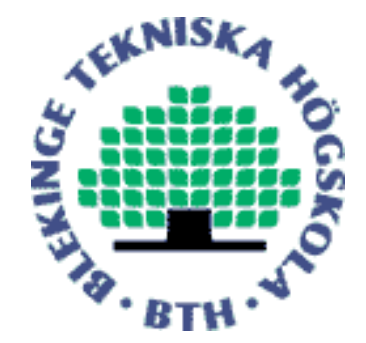

Copyright (C) 2011 IEEE.

Citation for the published paper:

Risk identification and risk mitigation instruments for global software development: Systematic review and survey results

Indira Nurdiani, Ronald jabangwe, Darja Smite, Daniela Damian

6th IEEE International Conference on Global Software Engineering Workshops, ICGSE

2011 Helsinki

This material is posted here with permission of the IEEE. Such permission of the IEEE does not in any way imply IEEE endorsement of any of BTH's products or services Internal or personal use of this material is permitted. However, permission to reprint/republish this material for advertising or promotional purposes or for creating new collective works for resale or redistribution must be obtained from the IEEE by sending a blank email message to pubs-permissions@iee.org.

By choosing to view this document, you agree to all provisions of the copyright laws protecting it. 


\title{
Risk Identification and Risk Mitigation Instruments for Global Software Development: Systematic Review and Survey Results
}

\author{
Indira Nurdiani*, Ronald Jabangwe ${ }^{\dagger}$, Darja Šmite ${ }^{\dagger}$, Daniela Damian* \\ * Software Engineering Global interAction Lab, University of Victoria, Victoria, Canada \\ ${ }^{\dagger}$ School of Computing, Blekinge Institute of Technology, Karlskrona, Sweden \\ *indiran@uvic.ca,danielad@cs.uvic.ca \\ $\dagger\{$ ronald.jabangwe, darja.smite\}@bth.se
}

\begin{abstract}
In this research we conducted a systematic review on Global Software Development (GSD) literature and performed a survey to gather challenges associated with GSD projects as well as their mitigation strategies. We reviewed studies with empirical evidence in GSD. In total there are 86 papers included as the primary studies. From the systematic review we collected 48 challenges and 42 mitigation strategies. These challenges and strategies were also identified by our survey respondents. In addition, 4 additional mitigation strategies were uncovered from the survey. The collected challenges and mitigation strategies are later compiled into checklists which can be employed as risk identification and risk mitigation instruments.
\end{abstract}

Keywords-risk identification; risk mitigation; systematic reviews; survey;

\section{INTRODUCTION}

The wave of business globalization has influenced how we develop software. Developing software is no longer just about engineers tied to their workstations in one collocated site. Nowadays, software developing organizations have adapted Global Software Development (GSD) with distributed development sites [19]. GSD promises certain benefits that attract software organization to venture into it, such as improving time to market, "round-the-clock" development, proximity to market, and access to cheaper yet skilled labor [3].

Nevertheless the benefits come with challenges due to the distance aspects, known as geographical distance, temporal distance (time zone difference), and socio-culture distance. Communication, coordination, and control are the processes that often suffer from the additional distance aspects. Pervasiveness of numerous challenges in GSD makes it a risky venture and more challenging [3], [10], [15].

Developing software in GSD settings requires additional effort in terms of project management, thus it affects the risk management process [15], [18]. When a company ventures into GSD the organization faces different constraints compared to collocated setting. Therefore risk management needs to be adjusted to the nature of GSD, because it posses unique characteristics and challenges [1], [13].

Risk Management is key to a successful business or project [6], including software development projects. The main part of risk management is risk identification and selecting the appropriate risk mitigation strategies [6]. Risk identification in GSD is deemed to be more difficult due to limited knowledge of challenges associated with GSD, even by the most experienced project managers [13].

In this paper we present the result of our study in identifying GSD challenges and strategies. We refer to challenges as issues or obstacles or circumstances that demand great consideration as they can negatively affect the outcome of a project. Risk is the probability of suffering loss while pursuing goals due to factors that are unpredictable or beyond [14]. Strategy is any activity or tactic employed to alleviate the impact or the occurrence of risks associated with challenges or problem. The sets of challenges and mitigation strategies were developed through the aid of a Systematic Literature Review (SLR) of empirical studies in GSD and industrial survey. The idea of this research is aligned with Šmite's proposal of risk management framework that contains GSD related risk identification checklists and accumulation of best practices that serves as knowledge base for risk mitigation guidance [20].

We organize the paper as follows. We discuss related work in Section II Section III describes the methodology we performed in the study to develop challenges and mitigation strategies. We present the results from SLR, survey and interview in Section IV and discussion in Section V. The paper is concluded in Section VI

\section{RELATED WORK}

One of the earlier work of software risk management was introduced by Barry Boehm [2]. He emphasized the importance of risk management in software development for the following reasons: avoiding disaster, avoiding rework, avoiding overkill, and stimulating win-win solutions. GSD has affected the way software is developed. It requires different kinds of structure compared to collocated development, including its risk management process. Risk management process not only needs to be tailored to GSD setting, but it also becomes more important in distributed software development projects than collocated project [18]. 
Risk management in GSD also needs to be performed in different levels, strategic, tactical, and operational. In strategic and tactical level, risk management is required to aid decision making whether to distribute the project or not. At the operational level risk management directly relates to software development processes and normally it is performed by project manager [17]. Prikladnicki et al [16] performed a Systematic Literature Review (SLR) to uncover patterns of GSD process model. From the study they uncovered five steps to minimize risks, they are: building trust, assessing offshore partner capabilities, prove operational efficiencies, enhance effectiveness, reengineer offshore partner into a more responsive development.

A conceptual framework for risk identification and mitigation for Scrum in GSD is proposed by Hossain et al [7]. The framework contains a list of risks and their possible mitigation strategies for implementing Scrum in GSD. A process model for distributed risk management is proposed by Kajko-Mattsson et al [8]. Their model emphasizes on process phases, roles in risk management, communication channels, and coordination actions. The model was based on an empirical study at IBM Sweden.

There are also other similar studies which are based on SLR. Lopez et al conducted an SLR to gather challenges and strategies for requirements engineering (RE) process in GSD [12]. A more recent work by Da Silva et al described a similar SLR to gather challenges and mitigation strategies in GSD and they also proposed a model for project management [4]. Both of these reviews entail identifying challenges and strategies in GSD with narrowed search on RE [12] and project management [4]. The systematic review we performed used broader search string and focused to study GSD challenges and mitigation strategies for risk identification and risk mitigation processes in GSD.

Existing works show that risk management needs to be done in different levels and at operational level it is usually done by project manager. In this study we uncovered a link between a person's role in the project with the challenges that he/she might encounter. Our study corroborates and further enforces previous study by Lane and Ågerfalk [11]. Also our study reveals a link between a person's role and the strategies that he/she implements that provides new insights in developing risk mitigation plan. Risk management needs to involve all team members and not just the project manager. The project manager might not encounter certain challenges the other team members with different roles encounter. We believe that the result of this study could serve as an initial step for further investigation of roles (developer, tester, project manager, etc) in software development processes, particularly risk management.

\section{Methodology}

A series of steps were taken during the development of the set of challenges and mitigation strategies. We kicked-off the study with an SLR by adopting the guidelines from Kitchenham and Charters [9]. Next step we identified additional challenges and strategies through a survey with industrial practitioners. Lastly we conducted follow-up inquiry with several respondents to seek feedback about the possibility of adopting the compiled sets of challenges and strategies as instruments for risk identification and risk mitigation as part of risk management process.

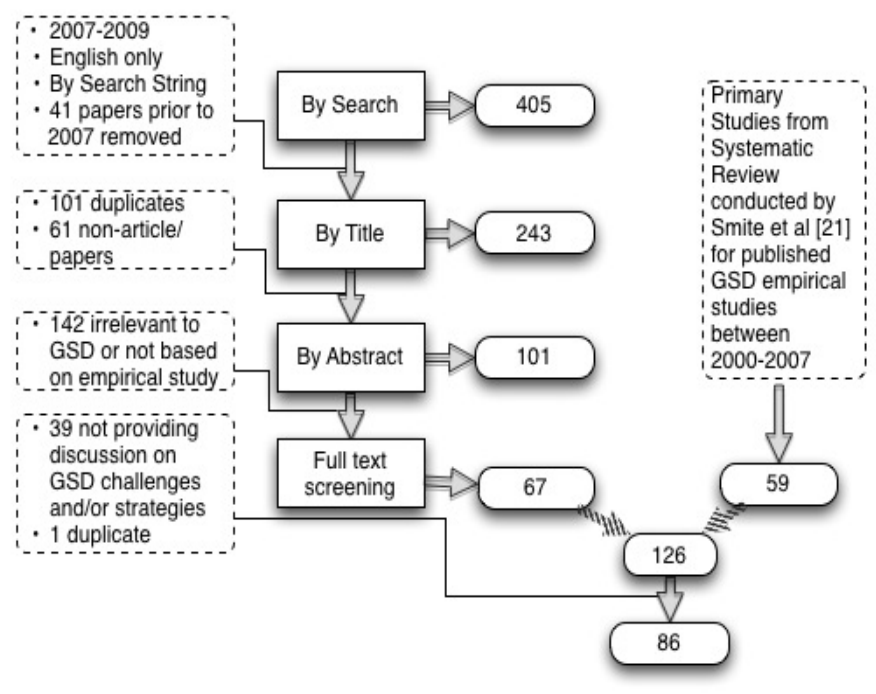

Figure 1. Systematic Review Steps

\section{A. Systematic Literature Review}

We collected GSD related challenges and mitigation strategies from empirical studies in GSD published between 2000 and 2009. Śmite et al had conducted a systematic review in the related subject area for published empirical studies between 2000 and 2007 [21]. We adopted similar search strategy conducted by Šmite et al in [21] to obtain empirical studies in GSD published between 2007 and 2009. In the review process, 67 papers published between 2007 and 2009 and 59 primary studies from the SLR conducted by Šmite et al were included in our full text screening. Later we only included studies that discuss challenges and strategies in GSD.

The SLR was conducted in December 2009. We searched for papers from seven main academic databases, Compendex, IEEE Xplore, Springer Link, ISI Web of Knowledge, ScienceDirect, Wiley Inter Science Journal Finder, and ACM Digital Library. We reviewed the full text of papers published between 2007-2009 and combined them with 59 papers from the primary studies included in [21]. Papers prior to 2000 were not included as GSD became an established area of research in the 21st century [21]. In total we reviewed 125 full text papers and included 86 papers as our primary studies 1 . Figure 1 shows the review steps that we performed.

\footnotetext{
${ }^{1}$ The complete list of primary studies and SLR findings is available online: http://home.segal.uvic.ca/ indiran/SLR/SLR_2007.htm
} 


\section{B. Survey}

In this research we also performed a survey to identify additional challenges and mitigation strategies that were not reported in the literature. Our survey targeted industrial practitioners who are or have been involved directly with distributed software development. They could be project managers, developer, testers, etc. We used convenient sampling in our survey. The survey was distributed through our immediate industrial contacts whom we knew are or have been working in distributed software development and later we asked them to forward and distribute it to their colleagues.

Table I

SURVEY OUTLINE

\begin{tabular}{|c|c|}
\hline Section in the survey & Description/Purpose \\
\hline General Information & $\begin{array}{l}\text { A brief description of the project they are } \\
\text { or were involved in i.e. their roles and } \\
\text { responsibilities in the past or current dis- } \\
\text { tributed software and also years of working } \\
\text { experience in GSD projects }\end{array}$ \\
\hline Project Characteristics & $\begin{array}{l}\text { Distribution characteristics of the project i.e: } \\
\text { use of different language, inter or intra- } \\
\text { company collaboration, geographical sepa- } \\
\text { ration, time zone difference. }\end{array}$ \\
\hline $\begin{array}{l}\text { Identifying Challenges \& } \\
\text { Strategies (separated into } \\
\text { subsections of challenges } \\
\text { and strategies for com- } \\
\text { munication, coordination, } \\
\text { and control process) }\end{array}$ & $\begin{array}{l}\text { The respondents were asked to identify } \\
\text { challenges that they faced in the project as } \\
\text { well as strategies that they implement in the } \\
\text { project from the sets that we identified from } \\
\text { the SLR. In the end of each subsections re- } \\
\text { spondents are asked to add other challenges } \\
\text { and strategies that are not listed. }\end{array}$ \\
\hline
\end{tabular}

Table I provides the outline of our survey. In the survey we enquire about general demographic information of the respondents, followed by a section to obtain information about the project characteristics. We also listed the challenges and strategies that we identified from the SLR. Respondents can select using checkboxes which challenges and strategies they currently face or practice according to their situation and also add additional challenges and strategies which are not included in the list.

\section{Follow-up Inquiries}

The subsequent step in our research is to explore the possibility of adopting the checklists as instruments to aid risk identification and mitigation process. Also we would like to know whether our respondents were aware with the challenges and strategies we mentioned in the checklists. All 13 respondents were asked to take part in the follow-up inquiry however due to time limitations we were only able to obtain 9 responses.

The follow-up inquiry was conducted through the aid of emails and instant messaging tools. Due to time limitations and heavy work load that some of the participants had at their jobs we were unable to conduct phone interviews. Also due to geographical distance we could not perform face-to- face interviews. Our respondents were dispersed in different countries in Europe, Australia, and Asia.

\section{RESUlts}

From the SLR we identified 48 challenges and 42 mitigation strategies. Taking into account the distance aspects in GSD and how each puts constraints on communication, coordination, and control processes, we categorized the challenges and mitigation strategies into different processes, where for each process the challenges are put into sub categories according to the distance aspects that influence them. The main categories are communication, coordination, and control. Each category has sub categories of Geographical Distance, Temporal Distance, and Socio-Culture distance. We followed systematic typological analysis to categorize the challenges and mitigation strategies [5].

Meanwhile from the survey, we received 31 responses however only 13 were completed. The respondents comprised of people from various organizations with different roles and working experience (ranging from 1 year to over than 10 years). The distribution of respondents and their roles are as follows: Project Manager (2), Project Coordinator (1), Functional Team Lead (1), Integration and Acceptance Engineer (1), Programmer/Developer (5), Technical Lead (2), Tester (1).

From the survey we did not identify additional challenges from our respondents. All 48 challenges were identified by at least one of the respondents as challenges they currently face or have in the past encountered. On the other hand we found 4 additional mitigation strategies from our respondents which were not identified from the SLR. The challenges which we compiled from the SLR and the survey are shown in Table II Challenges that can be categorized in different categories are in italics. The mitigation strategies which we collected from the SLR and the survey are compiled in Table III The additional strategies from our respondents are marked with asterisks.

In our survey results we found some challenges that are more commonly reported by certain roles. For instance, lack of team cohesiveness is reported by 3 (out of 5) developers, and one project manager. Challenges such as, legal issues and tailoring organization structure were reported by two respondents with technical lead roles. Some challenges, like cultural diversity and ensuring awareness, are identified by most respondents.

We also found a similar pattern with the strategies. We observed that there is a link between a person's role and the strategies that they implement in their situation. Some strategies, such as communicating expectations and evaluating remote partners capability, are identified by respondents with project manager or lead roles (one team lead and two project managers). It is worth noting that some strategies like using common communication language is reported by most of the respondents. 
Table II

GSD CHALLENGES FOR RISK IDENTIFICATION

\begin{tabular}{|c|c|}
\hline \multicolumn{2}{|r|}{ Communication } \\
\hline Temporal Distance & $\begin{array}{l}\text { Lack of synchronous communication } \\
\text { Delayed feedback }\end{array}$ \\
\hline Geographical Distance & $\begin{array}{l}\text { Lack of team cohesiveness } \\
\text { Communication effort overhead } \\
\text { Lack of Trust } \\
\text { Limited face-to-face meeting } \\
\text { Reduced communication richness or quality } \\
\text { Reduced possibility of informal communication } \\
\text { Extra travelling cost } \\
\text { Restricted inter-personal relationship build-up } \\
\text { Data loss during transfer } \\
\text { Reduced communication frequency }\end{array}$ \\
\hline Socio-Culture Distance & $\begin{array}{l}\text { Language difference } \\
\text { Different terminology usage between sites } \\
\text { Cultural diversity } \\
\text { Lack of mutual or shared understanding }\end{array}$ \\
\hline \multicolumn{2}{|r|}{ Coordination } \\
\hline Temporal Distance & Limited or no overlapping work hour \\
\hline Geographical Distance & $\begin{array}{l}\text { Ensure transparency of roles and responsibilities } \\
\text { Ensure awareness of changes to project artifacts } \\
\text { Define suitable software development process } \\
\text { Incompatible tools across sites } \\
\text { Appropriate tools are unavailable or insufficient } \\
\text { Limited working experience in GSD } \\
\text { Limited experience working together as a team } \\
\text { Properly define project scope } \\
\text { Ensure equal access to information } \\
\text { Plan appropriate activities distribution } \\
\text { Ensure appropriate information flow } \\
\text { Define appropriate change management process }\end{array}$ \\
\hline Socio-Culture Distance & $\begin{array}{l}\text { Insufficient knowledge and information sharing } \\
\text { Uncommon understanding in ways of working } \\
\text { Define, implement, and manage joint processes }\end{array}$ \\
\hline \multicolumn{2}{|r|}{ Control } \\
\hline Temporal Distance & $\begin{array}{l}\text { Plan appropriate activities distribution } \\
\text { Delayed feedback }\end{array}$ \\
\hline Geographical Distance & $\begin{array}{l}\text { Align stakeholders' goals and interests } \\
\text { Prepare accurate effort estimation } \\
\text { Maintain stakeholders' commitment } \\
\text { Problematic work item integration } \\
\text { Manage team dependencies } \\
\text { Tailor appropriate organizational structure } \\
\text { Differences of legal matter in different countries } \\
\text { Minimize fear of job loss } \\
\text { Maintain progress and status visibility } \\
\text { Security breach } \\
\text { Manage project's artefacts } \\
\text { Avoid rework of developed work items } \\
\text { Manage cost inflation }\end{array}$ \\
\hline Socio-Culture Distance & $\begin{array}{l}\text { Ensure equal domain knowledge } \\
\text { Minimize and manage staff turnover } \\
\text { Inadequate skill set }\end{array}$ \\
\hline
\end{tabular}

In our follow up inquiry, two respondents stated that the challenges and strategies sets contain certain challenges and practices that they were not aware of before. Thus, participating in our study helped them to be more aware of GSD related challenges and mitigation strategies. In addition
Table III

GSD StRategies For Risk Mitigation

\begin{tabular}{|c|c|}
\hline \multicolumn{2}{|r|}{ Communication } \\
\hline Temporal Distance & $\begin{array}{l}\text { Shift work hour } \\
\text { Reduce Communication hierarchy }\end{array}$ \\
\hline Geographical Distance & $\begin{array}{l}\text { Frequent travel } \\
\text { Implement communication model } \\
\text { Use groupware application } \\
\text { Promote frequent communication } \\
\text { Use rich communication media }\end{array}$ \\
\hline Socio-Culture Distance & $\begin{array}{l}\text { Appoint a liaison } \\
\text { Develop a special terminology dictionary } \\
\text { Use a common communication language } \\
\text { Team rotation among development sites } \\
\text { Team building exercises during cross site visit }\end{array}$ \\
\hline \multicolumn{2}{|r|}{ Coordination } \\
\hline Temporal Distance & Establish buddy system \\
\hline Geographical Distance & $\begin{array}{l}\text { Distribute task based on architectural decoupling } \\
\text { Promote frequent work item deliveries } \\
\text { Use configuration management tool } \\
\text { Create directory listing of team members with } \\
\text { their expertise \& responsibility } \\
\text { Face-to-face kick off meeting } \\
\text { Nearshoring } \\
\text { Minimize number distributed development sites } \\
\text { Ensure compatibility and consistency of tools } \\
\text { Create a project structure that includes tasks \& } \\
\text { artifacts dependencies } \\
\text { Maintain and share project artefacts repository } \\
\text { Perform joint requirements analysis } \\
\text { Temporary collocation during critical phases }\end{array}$ \\
\hline Socio-Culture Distance & $\begin{array}{l}\text { Evaluate team members assigned to the project } \\
\text { Evaluate remote partner capability }\end{array}$ \\
\hline \multicolumn{2}{|r|}{ Control } \\
\hline $\begin{array}{l}\text { Temporal Distance } \\
\text { Geographical Distance }\end{array}$ & $\begin{array}{l}\text { Frequent synchronous communication } \\
\text { Establish communication guidelines } \\
\text { Build consensus on working norms } \\
\text { Agree on cost and effort estimation } \\
\text { Build "experience factory" } \\
\text { Secure intellectual property } \\
\text { Consider inflation rate in different countries } \\
\text { Promote appropriate level of autonomy } \\
\text { Tailor communication \& coordination to project } \\
\text { changes } \\
\text { Communicate expectations \& rules early } \\
\text { Frequent project status update } \\
\text { Perform regular quality audit* } \\
\text { Communicate quality assurance guidelines* } \\
\text { Manage employee contract* }\end{array}$ \\
\hline Socio-Culture Distance & $\begin{array}{l}\text { Acknowledge cultural difference } \\
\text { Ensure upper management support through the } \\
\text { project } \\
\text { Apply appropriate rewards to employees } \\
\text { Provide Training } \\
\text { Cross-skilling* }\end{array}$ \\
\hline
\end{tabular}

all of our respondents mentioned that they will consider using the checklists for future GSD projects. One respondent added that for future use the checklists require adjustments according to the project environment. 


\section{Discussion}

We developed two checklists that could be utilized in identifying challenges and strategies in GSD projects. All 48 challenges and 42 mitigation strategies mentioned in the checklists were identified by the survey respondents. Consequently this illustrates a match between GSD empirical studies included in our SLR and what is being reported by industry practitioners. It shows that GSD researchers are on the right track in identifying challenges and strategies perceived by industry, as shown from the survey results.

Even though the data in the checklists is categorized in a particular order, some of the challenges and mitigation strategies can fall in a different category as well. One challenge can be an issue in coordination as well as in control. For example the issue surrounding lack of process transparency and progress visibility is a challenge in both control and coordination mechanisms. In such cases we referred to the papers to identify the context that the challenge or practice was being discussed.

GSD is highly situational, one challenge can lead to different risks depending on the organizations context [21]. The same applies for the relation between challenges and selecting appropriate strategies. In this study we did not perform correlation analysis between challenges and risks, nor between challenges and strategies. We cannot provide prescription of translating challenges into risks as well as selecting appropriate strategies from our preliminary study. Thus, the checklists should be used more as a foundation for identifying challenges and the appropriate mitigation strategies in a particular situation. This was also stated by one respondent who mentioned that they would use the checklists as a baseline for developing their own checklists that would be more relevant to their own situation.

Risk identification in risk management processes is considered to be more difficult in GSD projects. It is due to limited knowledge regarding GSD settings. As mentioned by Moe and Šmite, even the most experienced project managers can fail in GSD projects [13]. Therefore the checklists can also be incorporated as instruments in risk management processes, specifically as a foundation in identifying risk and developing risk mitigation plans.

One's perception of the prevalence of a challenge in certain settings is closely related to their roles and responsibilities in that project, this finding corroborates with the study reported by Lane and Ågerfalk [11]. Although we cannot provide statistical proof, we can observe some challenges that are encountered by certain team members, may not be relevant for other team members in a GSD project. It implies that there is a link between a person's role and the challenges that he/she encounters. Similar pattern was also found with the mitigation strategies. Taking this finding into account we recommend the involvement of team members with different roles in the risk management process in a form of cross-functional team.

\section{CONCLUSiOn AND Future Work}

All 48 challenges and 42 mitigation strategies uncovered through the systematic literature were identified by our survey respondents. Therefore it was concluded that all the challenges and strategies that were collected during the systematic literature review were prevalent in certain industrial settings. This does not mean that every GSD setting experiences each and every challenge and strategy uncovered. However, this shows that the challenges are a factor in certain GSD scenarios and the strategies have been or are currently being utilized in certain GSD settings as well.

Furthermore there were 4 additional strategies that were found from the survey. However we did not identify additional challenges from the survey. The absence of new challenges in the questionnaire did not merit a conclusion that all possible challenges that can be encountered in GSD have all been revealed and discussed in literature. There is a possibility that the respondents may have not been aware of other challenges. Also it is also important to note that the number of respondents could be a factor in the results as well. The possibility that more challenges and practices could have been collected had the number of respondents been significantly higher should not be ruled out.

Furthermore from the survey results, it can be seen that there is a connection between a persons role in a project and the challenges he or she faced. This interesting phenomenon has also been identified and addressed in [11]. However, from the primary studies and other supporting studies included in this research, the connection between a person's role in a project and the mitigation strategies that he or she practiced or is aware of, has not yet been addressed in literature.

For future work, it is important to implement the checklist in an industrial setting to identify GSD related challenges and mitigation strategies. More information can then be obtained on the usability and the usefulness of the checklist. Furthermore investigation on why several challenges are not encountered and also why certain strategies are not applicable in certain environment can be revealed. The results of such studies can also be used towards the development of decision making mechanism to mitigate GSD related challenges.

The developed GSD challenges and mitigation strategies are static checklists. The checklists can be used as a base for risk identification and risk mitigation planning in a distributed software development setting. However they cannot be used as decision making tool to select the most appropriate strategies for particular challenges. Thus for future work the collected challenges and mitigation strategies can be prioritized to aid decision making process in selecting the most suitable mitigation strategies for particular challenges. 
The prioritization needs to take into account the variety of GSD settings and scenarios.

\section{ACKNOWLEDGMENT}

This research, including the survey and interview, took place during the first and second author's master thesis project at Blekinge Institute of Technology, Sweden between December 2009 and May 2010 under supervision of Dr. Darja Šmite. We would also like to thank our colleagues at SEGAL for their valuable input to improve this paper.

\section{REFERENCES}

[1] W. Aspray, F. Mayadas, and M. Vardi, editors. Globalization and Offshoring of Software - A Report of the ACM Job Migration Task Force. Association for Computing Machinery, 2006.

[2] B. Boehm. Software Risk Management. IEEE Computer Society Press, Washington, DC, United states, 1989.

[3] E. Carmel. Global software teams: collaborating across borders and time zones. Prentice Hall PTR, 1999.

[4] F. Q. da Silva, C. Costa, A. C. C. Franca, and R. Prikladinicki. Challenges and solutions in distributed software development project management: A systematic literature review. Global Software Engineering, International Conference on, 0:87-96, 2010.

[5] L. M. Given. SAGE Encyclopedia of Qualitative Research Methods, volume 1\&2. SAGE, USA, 2008.

[6] D. Hillson and R. Murray-Webster. Understanding and managing risk attitude. Gower, Aldershot, 2nd edition, 2007.

[7] E. Hossain, M. A. Babar, H. young Paik, and J. Verner. Risk identification and mitigation processes for using scrum in global software development: A conceptual framework. AsiaPacific Software Engineering Conference, 0:457-464, 2009.

[8] M. Kajko-Mattsson, K. Sjokvist, J. Soderstrom, and D. Krogdahl. Drimap-a model of distributed risk management process. In INC, IMS and IDC, 2009. NCM '09. Fifth International Joint Conference on, pages $994-1000$, August 2009.

[9] B. Kitchenham and S. Charters. Guidelines for performing systematic literature reviews in software engineering. Version, 2:2007-01, 2007.

[10] M. Korkala and P. Abrahamsson. Communication in distributed agile development: A case study. In 33rd EUROMICRO Conference on Software Engineering and Advanced Applications, SEAA 2007, August 27, 2007 - August 31, 2007, pages 203-210, Lubeck, Germany, 2007. Inst. of Elec. and Elec. Eng. Computer Society.
[11] M. Lane and P. Agerfalk. On the suitability of particular software development roles to global software development. 2008 3RD IEEE INTERNATIONAL CONFERENCE GLOBAL SOFTWARE ENGINEERING,, pages 3-12, 2008.

[12] A. Lopez, J. Nicolas, and A. Toval. Risks and safeguards for the requirements engineering process in global software development. In Proceedings - 2009 4th IEEE International Conference on Global Software Engineering, ICGSE 2009, pages 394 - 399, Limerick, Ireland, 2009.

[13] N. B. Moe and D. Smite. Understanding a lack of trust in global software teams: A multiple-case study. Software Process Improvement and Practice, 13(3):217-231, 2008.

[14] C. R. Pandian. Applied software risk management. CRC Press, Dec. 2006.

[15] R. Prikladnicki. Global software development in practice lessons learned. Software process improvement and practice, 8(4):267, 2003.

[16] R. Prikladnicki, J. Audy, and F. Shull. Patterns in effective distributed software development. Software, IEEE, 27(2):12 -15, March-April 2010.

[17] R. Prikladnicki, J. R. Evaristo, J. L. N. Audy, and M. H. Yamaguti. Risk management in distributed it projects: Integrating strategic, tactical, and operational levels. IJeC, 2(4):1-18, 2006.

[18] R. Prikladnicki and M. Yamaguti. Risk management in global software development: a position paper. In "Third International Workshop on Global Software Development (GSD 2004) - 26th International Conference on Software Engineering, pages 18 - 20, Stevenage, UK, 2004.

[19] R. Sangwan, M. Bass, N. Mullick, D. J. Paulish, and J. Kazmeier. Global Software Development Handbook (Auerbach Series on Applied Software Engineering Series). Auerbach Publications, 2006.

[20] D. Šmite. Project outcome predictions: Risk barometer based on historical data. In International Conference on Global Software Engineering, ICGSE 2007, August 27, 2007 - August 30, 2007, pages 103-112, Munich, Germany, 2007. Inst. of Elec. and Elec. Eng. Computer Society.

[21] D. Šmite, C. Wohlin, T. Gorschek, and R. Feldt. Empirical evidence in global software engineering: a systematic review. Empirical Software Engineering, 2009. 